\title{
Liquid Crystals
}

\section{Crystal structure of a cholesterol-based dimesogen}

\author{
M. A. Sridhar , N. K. Lokanath , J. Shashidhara Prasad , C. V. Yelammagad \& S. \\ K. Varshney
}

To cite this article: M. A. Sridhar , N. K. Lokanath, J. Shashidhara Prasad, C. V. Yelammagad \& S. K. Varshney (2001) Crystal structure of a cholesterol-based dimesogen, Liquid Crystals, 28:1, 45-49, DOI: $10.1080 / 02678290010002201$

To link to this article: https://doi.org/10.1080/02678290010002201

冓 Published online: 06 Aug 2010.

Submit your article to this journal

山 Article views: 59

Q View related articles $\sqsubset$

4 Citing articles: 9 View citing articles ๘ 


\title{
Crystal structure of a cholesterol-based dimesogen
}

\author{
M. A. SRIDHAR, N. K. LOKANATH, J. SHASHIDHARA PRASAD* \\ Department of Studies in Physics, University of Mysore, Mysore 570006, India
}

C. V. YELAMMAGAD and S. K. VARSHNEY

Center for Liquid Crystal Research, P.B. No. 1329, Jalahalli,

Bangalore 560013, India

(Received 14 January 2000; in final form 27 June 2000; accepted 27 June 2000)

\begin{abstract}
The title compound, cholesteryl 6[4-(4-pentyloxyphenylethynyl)phenoxy]hexanoate (DMT5), a cholesterol-based dimesogen, crystallizes in the monoclinic space group $P 2_{1}$ with the parameters $a=9.812(3) \AA, b=9.713(2) \AA, c=24.179(2) \AA, \beta=92.48(1)^{\circ} V=2302.2(9) \AA^{3}, Z=2$, $F_{000}=836, \lambda\left(\mathrm{MoK}_{\alpha}\right)=0.71069 \AA, \mu=0.067 \mathrm{~cm}^{-1}$, final $R=0.0772$. The structure has intermolecular hydrogen bonds; it is stabilized by the presence of intermolecular contacts whose spacings are less than van der Waals' radii.
\end{abstract}

\section{Introduction}

Liquid crystalline dimers consisting of two identical mesogenic units connected linearly via a flexible spacer, such as polymethylene or oligosiloxyl group, are well known [1]. Compounds in which the chemical structures of the two mesogenic units are different have also been studied [2]. Such compounds, also called dimesogens, have been reported to exhibit an interesting polymorphic sequence including incommensurate smectic phases [3]. We previously synthesized a dimesogen referred to as DMT4, which has a cholesteryl ester unit and tolane moiety connected by a polymethylene $\left(\mathrm{C}_{5}\right)$ spacer. It shows just a single mesophase-a cholesteric phase having a wide temperature range of about $130^{\circ}$ [4]. To get a better understanding of the effect of the length of the side chain on the mesophase, we have synthesized a number

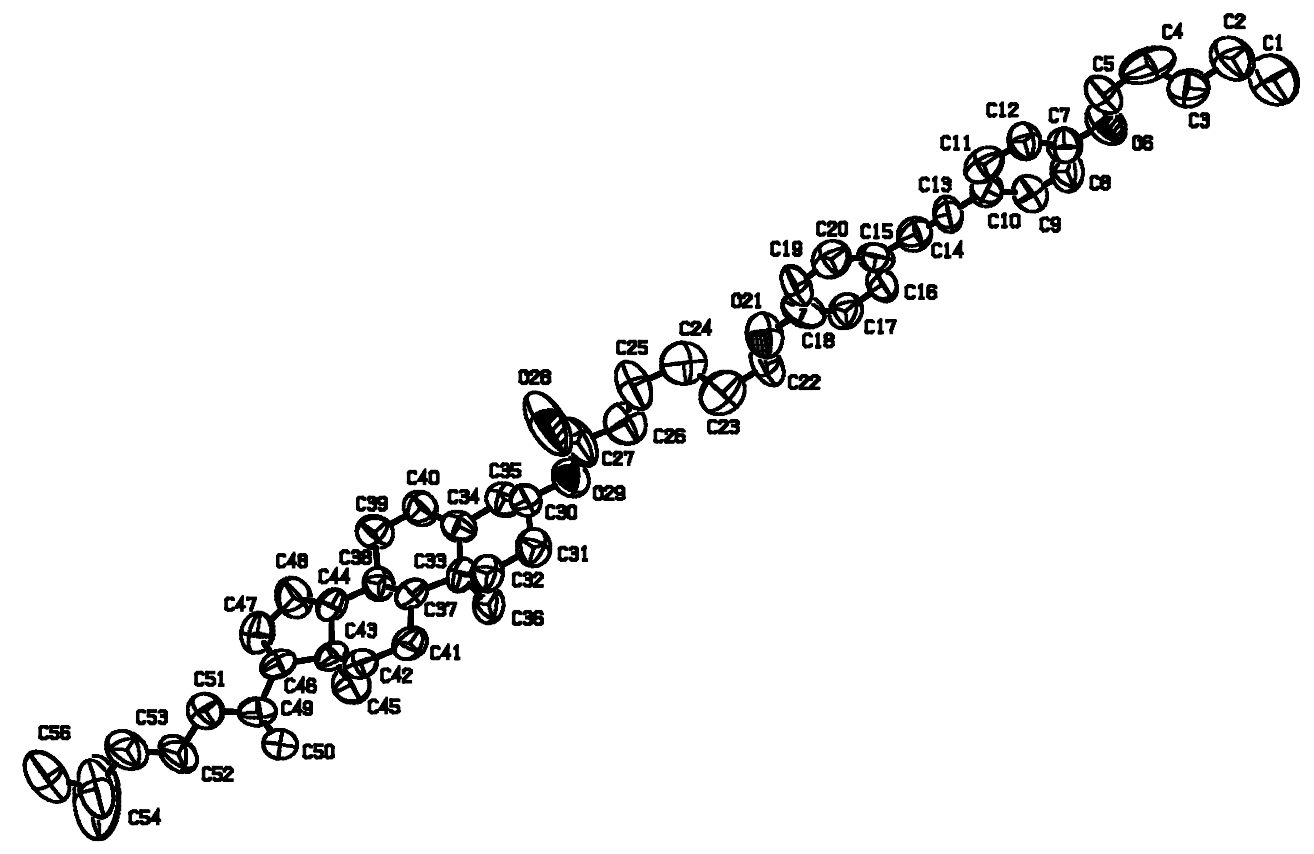

Figure 1. ORTEP of the DMT5 molecule at 50\% probability.

*Author for correspondence, e-mail: mysxrd@giasbg01.vsnl.net.in 
of higher and lower homologues of DMT4. Owing to their exotic nature and properties, it will be interesting to look at the crystal structure of these dimesogens. In this paper we report the crystal structure of one of the homologues of DMT4, namely cholesteryl 6[4-(4-pentyloxyphenylethyn yl)phenoxy] hexanoate, DMT5 (figure 1). This compound melts to the cholesteric phase at $124.8^{\circ} \mathrm{C}$ and goes to the isotropic phase at $199.3^{\circ} \mathrm{C}$. It is evident that this homologue has a greatly reduced mesophase range as compared with DMT4.

\section{Experimental}

The crystals were colourless. A sample of size $0.3 \times 0.3 \times 0.5 \mathrm{~mm}^{3}$ was selected for data collection. The crystal was mounted on a glass fibre and the data collection was carried out on an Enraf-Nonius CAD4 single crystal X-ray diffractometer with graphite monochromated $\mathrm{MoK}_{\alpha}$ radiation. Data were collected upto $2 \theta=50^{\circ}$. The index ranges are as follows: $-11 \leqslant h \leqslant 11$, $0 \leqslant k \leqslant 11,0 \leqslant l \leqslant 28$. A total of 4308 reflections were collected of which 1597 has $I \geqslant 2 \sigma(I)$. Lorentz and polarization corrections were applied. The structure was solved by direct methods (SHELXS-97 [5]). Structure was revealed in the first map; it was refined (SHELXL-97

Table 1. Crystal data and structure refinement details.

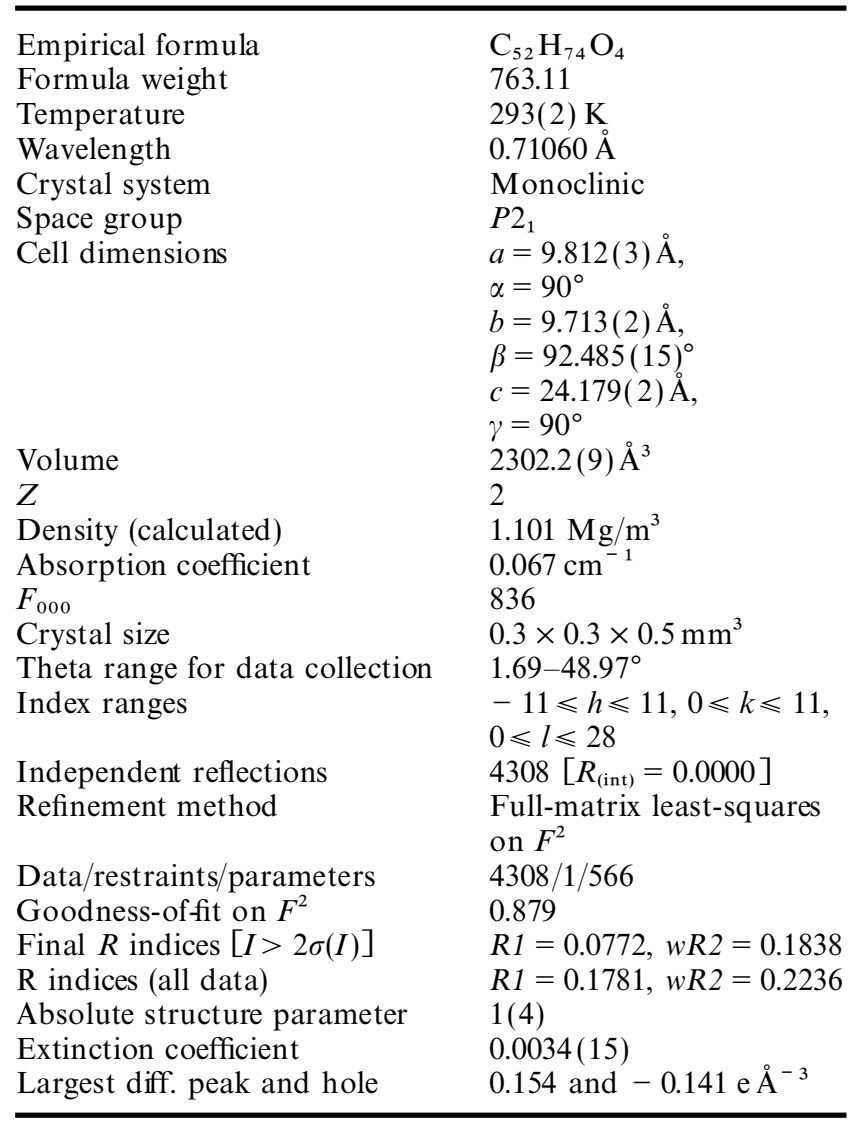

Table 2. Atomic coordinates and equivalent isotropic temperature factors of non-hydrogen atoms.

\begin{tabular}{|c|c|c|c|c|}
\hline Atom & $X$ & $Y$ & $Z$ & $U_{\mathrm{eq}}$ \\
\hline $\mathrm{C} 1$ & $-0.7742(16)$ & $1.062(2)$ & $-0.2202(8)$ & $0.237(8)$ \\
\hline $\mathrm{C} 2$ & $-0.7192(13)$ & $0.9386(19)$ & $-0.2078(6)$ & $0.179(6)$ \\
\hline $\mathrm{C} 3$ & $-0.6081(12)$ & $0.9328(15)$ & $-0.1618(6)$ & $0.157(4)$ \\
\hline $\mathrm{C} 4$ & $-0.5484(13)$ & $0.8088(13)$ & $-0.1535(6)$ & $0.170(5)$ \\
\hline $\mathrm{C} 5$ & $-0.4370(10)$ & $0.8027(13)$ & $-0.1077(4)$ & $0.125(4)$ \\
\hline C6 & $-0.3401(7)$ & $0.9054(8)$ & $-0.1184(2)$ & $0.134(2)$ \\
\hline $\mathrm{C} 7$ & $-0.2164(11)$ & $0.8949(12)$ & $-0.0882(4)$ & $0.101(3)$ \\
\hline $\mathrm{C} 8$ & $-0.1220(14)$ & $0.9914(13)$ & $-0.1012(4)$ & $0.125(4)$ \\
\hline C9 & $0.0052(11)$ & $0.9946(12)$ & $-0.0778(4)$ & $0.111(3)$ \\
\hline $\mathrm{C} 10$ & $0.0423(11)$ & $0.8946(11)$ & $-0.0377(3)$ & $0.102(3)$ \\
\hline $\mathrm{C} 11$ & $-0.0524(13)$ & $0.7989(10)$ & $-0.0239(4)$ & $0.109(3)$ \\
\hline $\mathrm{C} 12$ & $-0.1851(10)$ & $0.7993(11)$ & $-0.0510(4)$ & $0.102(3)$ \\
\hline $\mathrm{C} 13$ & $0.1774(10)$ & $0.9001(10)$ & $-0.0136(3)$ & $0.105(3)$ \\
\hline $\mathrm{C} 14$ & $0.2896(10)$ & $0.9018(10)$ & $0.0086(4)$ & $0.102(3)$ \\
\hline $\mathrm{C} 15$ & $0.4212(10)$ & $0.9046(12)$ & $0.0338(4)$ & $0.099(3)$ \\
\hline $\mathrm{C} 16$ & $0.5069(11)$ & $1.0087(13)$ & $0.0201(4)$ & $0.105(3)$ \\
\hline $\mathrm{C} 17$ & $0.6379(11)$ & $1.0231(10)$ & $0.0458(4)$ & $0.104(3)$ \\
\hline $\mathrm{C} 18$ & $0.6757(11)$ & $0.9312(14)$ & $0.0862(5)$ & $0.120(4)$ \\
\hline C19 & $0.5936(12)$ & $0.8319(15)$ & $0.1011(4)$ & $0.122(4)$ \\
\hline $\mathrm{C} 20$ & $0.4678(13)$ & $0.8154(11)$ & $0.0765(5)$ & $0.118(3)$ \\
\hline $\mathrm{C} 21$ & $0.7971(7)$ & $0.9333(8)$ & $0.1172(3)$ & $0.130(2)$ \\
\hline $\mathrm{C} 22$ & $0.8902(10)$ & $1.0349(14)$ & $0.1078(4)$ & $0.135(4)$ \\
\hline $\mathrm{C} 23$ & $1.0097(12)$ & $1.0285(15)$ & $0.1504(5)$ & $0.153(4)$ \\
\hline $\mathrm{C} 24$ & $1.0868(11)$ & $0.9030(16)$ & $0.1429(5)$ & $0.157(4)$ \\
\hline $\mathrm{C} 25$ & $1.1944(11)$ & $0.8669(15)$ & $0.1903(5)$ & $0.159(5)$ \\
\hline $\mathrm{C} 26$ & $1.3072(10)$ & $0.9672(13)$ & $0.1974(4)$ & $0.139(4)$ \\
\hline $\mathrm{C} 27$ & $1.4130(14)$ & $0.928(2)$ & $0.2394(5)$ & $0.159(5)$ \\
\hline $\mathrm{C} 28$ & $1.4211(14)$ & $0.8202(16)$ & $0.2568(6)$ & $0.339(11)$ \\
\hline $\mathrm{C} 29$ & $1.4211(8)$ & $1.0148(8)$ & $0.2540(3)$ & $0.132(2)$ \\
\hline C30 & $1.5973(9)$ & $0.9881(10)$ & $0.2972(4)$ & $0.100(3)$ \\
\hline C31 & $1.5886(8)$ & $1.0988(10)$ & $0.3381(4)$ & $0.109(3)$ \\
\hline C32 & $1.6971(8)$ & $1.0783(9)$ & $0.3850(3)$ & $0.100(3)$ \\
\hline C33 & $1.8432(7)$ & $1.0749(8)$ & $0.3652(3)$ & $0.075(2)$ \\
\hline C34 & $1.8476(9)$ & $0.9747(10)$ & $0.3173(3)$ & $0.090(2)$ \\
\hline C35 & $1.7363(9)$ & $0.9890(11)$ & $0.2721(3)$ & $0.114(3)$ \\
\hline $\mathrm{C} 36$ & $1.8806(9)$ & $1.2183(9)$ & $0.3448(3)$ & $0.106(3)$ \\
\hline C37 & $1.9398(7)$ & $1.0284(7)$ & $0.4133(3)$ & $0.074(2)$ \\
\hline C38 & $2.0817(7)$ & $0.9828(9)$ & $0.3931(3)$ & $0.087(2)$ \\
\hline C39 & $2.0602(8)$ & $0.8616(10)$ & $0.3535(3)$ & $0.104(3)$ \\
\hline $\mathrm{C} 40$ & $1.9434(10)$ & $0.8808(11)$ & $0.3133(4)$ & $0.108(3)$ \\
\hline C41 & $1.9548(8)$ & $1.1289(9)$ & $0.4602(3)$ & $0.102(3)$ \\
\hline $\mathrm{C} 42$ & $2.0565(8)$ & $1.0879(8)$ & $0.5075(3)$ & $0.093(2)$ \\
\hline $\mathrm{C} 43$ & $2.1945(7)$ & $1.0555(7)$ & $0.4859(3)$ & $0.078(2)$ \\
\hline $\mathrm{C} 44$ & $2.1708(7)$ & $0.9437(8)$ & $0.4426(3)$ & $0.079(2)$ \\
\hline $\mathrm{C} 45$ & $2.2577(9)$ & $1.1872(9)$ & $0.4651(4)$ & $0.125(3)$ \\
\hline $\mathrm{C} 46$ & $2.2972(7)$ & $0.9790(8)$ & $0.5259(3)$ & $0.083(2)$ \\
\hline $\mathrm{C} 47$ & $2.3880(8)$ & $0.9013(11)$ & $0.4869(3)$ & $0.117(3)$ \\
\hline $\mathrm{C} 48$ & $2.3143(8)$ & $0.8920(13)$ & $0.4310(4)$ & $0.133(4)$ \\
\hline C49 & $2.3790(9)$ & $1.0554(10)$ & $0.5707(4)$ & $0.105(3)$ \\
\hline $\mathrm{C} 50$ & $2.2883(11)$ & $1.1408(10)$ & $0.6080(4)$ & $0.145(4)$ \\
\hline C51 & $2.4669(9)$ & $0.9595(11)$ & $0.6052(4)$ & $0.119(3)$ \\
\hline C52 & $2.5775(10)$ & $1.0217(11)$ & $0.6429(5)$ & $0.161(5)$ \\
\hline C53 & $2.6534(10)$ & $0.9159(13)$ & $0.6783(4)$ & $0.144(4)$ \\
\hline C54 & $2.7645(14)$ & $0.961(2)$ & $0.7167(6)$ & $0.201(7)$ \\
\hline C55 & $2.858(2)$ & $1.019(2)$ & $0.6885(12)$ & $0.42(2)$ \\
\hline C56 & $2.8197(12)$ & $0.837(2)$ & $0.7478(5)$ & $0.200(6)$ \\
\hline
\end{tabular}


Table 3. Anisotropic thermal parameters of the non-hydrogen atoms.

\begin{tabular}{|c|c|c|c|c|c|c|}
\hline Atom & $U_{11}$ & $U_{22}$ & $U_{33}$ & $U_{23}$ & $U_{13}$ & $U_{12}$ \\
\hline $\mathrm{C} 1$ & $0.186(15)$ & $0.25(2)$ & $0.270(18)$ & $0.004(16)$ & $-0.038(13)$ & $-0.023(19)$ \\
\hline $\mathrm{C} 2$ & $0.134(10)$ & $0.202(16)$ & $0.197(12)$ & $-0.001(11)$ & $-0.030(10)$ & $-0.026(13)$ \\
\hline $\mathrm{C} 3$ & $0.139(9)$ & $0.113(10)$ & $0.215(12)$ & $-0.015(8)$ & $-0.033(9)$ & $-0.011(9)$ \\
\hline $\mathrm{C} 4$ & $0.161(11)$ & $0.102(9)$ & $0.250(16)$ & $-0.043(10)$ & $0.067(11)$ & $-0.043(10)$ \\
\hline C5 & $0.102(7)$ & $0.164(10)$ & $0.106(7)$ & $-0.003(8)$ & $-0.026(6)$ & $-0.005(7)$ \\
\hline C6 & $0.113(5)$ & $0.169(7)$ & $0.120(5)$ & $-0.036(5)$ & $-0.013(4)$ & $0.026(5)$ \\
\hline C7 & $0.114(8)$ & $0.102(7)$ & $0.086(6)$ & $-0.003(7)$ & $0.004(6)$ & $0.010(6)$ \\
\hline $\mathrm{C} 8$ & $0.136(9)$ & $0.121(10)$ & $0.115(8)$ & $0.011(9)$ & $-0.016(8)$ & $0.035(7)$ \\
\hline C9 & $0.107(7)$ & $0.126(9)$ & $0.100(7)$ & $0.001(7)$ & $-0.007(6)$ & $0.003(7)$ \\
\hline $\mathrm{C} 10$ & $0.140(9)$ & $0.099(7)$ & $0.067(5)$ & $0.031(8)$ & $-0.004(6)$ & $-0.007(6)$ \\
\hline C11 & $0.152(10)$ & $0.080(6)$ & $0.097(7)$ & $-0.001(7)$ & $0.035(7)$ & $0.018(6)$ \\
\hline $\mathrm{C} 12$ & $0.097(7)$ & $0.108(8)$ & $0.100(7)$ & $-0.002(6)$ & $-0.001(6)$ & $0.019(7)$ \\
\hline $\mathrm{C} 13$ & $0.104(7)$ & $0.119(8)$ & $0.090(6)$ & $0.030(7)$ & $-0.018(6)$ & $0.003(6)$ \\
\hline $\mathrm{C} 14$ & $0.104(7)$ & $0.105(7)$ & $0.098(6)$ & $0.019(6)$ & $0.012(6)$ & $-0.011(5)$ \\
\hline $\mathrm{C} 15$ & $0.092(7)$ & $0.109(8)$ & $0.096(7)$ & $0.000(7)$ & $-0.005(6)$ & $-0.034(6)$ \\
\hline C16 & $0.102(7)$ & $0.123(9)$ & $0.088(7)$ & $0.021(7)$ & $-0.025(6)$ & $-0.011(6)$ \\
\hline $\mathrm{C} 17$ & $0.129(9)$ & $0.088(7)$ & $0.095(7)$ & $0.008(7)$ & $0.006(6)$ & $0.014(5)$ \\
\hline $\mathrm{C} 18$ & $0.083(7)$ & $0.152(11)$ & $0.124(8)$ & $-0.044(8)$ & $0.005(7)$ & $-0.015(8)$ \\
\hline $\mathrm{C} 19$ & $0.083(7)$ & $0.167(13)$ & $0.113(8)$ & $0.014(8)$ & $-0.034(7)$ & $0.007(8)$ \\
\hline $\mathrm{C} 20$ & $0.137(10)$ & $0.107(8)$ & $0.110(7)$ & $0.000(7)$ & $0.017(7)$ & $0.010(7)$ \\
\hline $\mathrm{C} 21$ & $0.084(4)$ & $0.151(6)$ & $0.153(6)$ & $-0.014(5)$ & $0.007(4)$ & $0.039(5)$ \\
\hline $\mathrm{C} 22$ & $0.086(7)$ & $0.194(12)$ & $0.122(8)$ & $0.020(8)$ & $-0.030(6)$ & $0.002(8)$ \\
\hline $\mathrm{C} 23$ & $0.151(10)$ & $0.154(11)$ & $0.157(10)$ & $0.016(10)$ & $0.049(8)$ & $-0.005(9)$ \\
\hline $\mathrm{C} 24$ & $0.136(9)$ & $0.165(12)$ & $0.170(11)$ & $-0.009(10)$ & $0.025(8)$ & $-0.013(10)$ \\
\hline $\mathrm{C} 25$ & $0.115(7)$ & $0.211(13)$ & $0.148(9)$ & $-0.005(9)$ & $-0.041(7)$ & $0.040(10)$ \\
\hline $\mathrm{C} 26$ & $0.122(8)$ & $0.152(10)$ & $0.142(9)$ & $-0.015(8)$ & $0.011(7)$ & $0.010(8)$ \\
\hline $\mathrm{C} 27$ & $0.142(10)$ & $0.195(15)$ & $0.134(8)$ & $-0.062(11)$ & $-0.049(8)$ & $0.081(10)$ \\
\hline $\mathrm{C} 28$ & $0.305(14)$ & $0.273(15)$ & $0.416(19)$ & $-0.173(13)$ & $-0.252(14)$ & $0.190(14)$ \\
\hline $\mathrm{C} 29$ & $0.116(5)$ & $0.118(6)$ & $0.160(6)$ & $-0.025(5)$ & $-0.034(5)$ & $0.015(5)$ \\
\hline $\mathrm{C} 30$ & $0.108(7)$ & $0.087(6)$ & $0.102(6)$ & $-0.003(6)$ & $-0.026(6)$ & $0.006(6)$ \\
\hline $\mathrm{C} 31$ & $0.082(6)$ & $0.116(8)$ & $0.130(7)$ & $0.003(5)$ & $0.006(5)$ & $0.008(7)$ \\
\hline $\mathrm{C} 32$ & $0.090(6)$ & $0.096(6)$ & $0.114(6)$ & $0.001(5)$ & $0.004(5)$ & $0.018(6)$ \\
\hline $\mathrm{C} 33$ & $0.068(5)$ & $0.065(5)$ & $0.092(6)$ & $0.002(4)$ & $0.016(4)$ & $0.023(5)$ \\
\hline C34 & $0.097(6)$ & $0.100(7)$ & $0.073(5)$ & $-0.009(6)$ & $0.001(5)$ & $-0.003(6)$ \\
\hline C35 & $0.118(7)$ & $0.121(8)$ & $0.101(6)$ & $0.007(7)$ & $-0.007(6)$ & $-0.014(6)$ \\
\hline C36 & $0.112(6)$ & $0.092(6)$ & $0.113(6)$ & $0.002(5)$ & $-0.014(5)$ & $0.031(5)$ \\
\hline $\mathrm{C} 37$ & $0.080(5)$ & $0.061(5)$ & $0.083(5)$ & $0.002(4)$ & $0.024(4)$ & $0.002(4)$ \\
\hline C38 & $0.073(5)$ & $0.086(6)$ & $0.100(6)$ & $-0.006(5)$ & $-0.002(5)$ & $0.012(5)$ \\
\hline C39 & $0.093(6)$ & $0.128(8)$ & $0.093(6)$ & $0.008(6)$ & $0.013(5)$ & $-0.023(6)$ \\
\hline $\mathrm{C} 40$ & $0.104(7)$ & $0.125(8)$ & $0.094(6)$ & $0.014(7)$ & $-0.006(6)$ & $-0.011(6)$ \\
\hline $\mathrm{C} 41$ & $0.095(6)$ & $0.097(6)$ & $0.116(7)$ & $0.022(5)$ & $0.013(5)$ & $-0.023(6)$ \\
\hline $\mathrm{C} 42$ & $0.099(6)$ & $0.078(6)$ & $0.102(6)$ & $0.006(5)$ & $-0.001(5)$ & $-0.019(5)$ \\
\hline $\mathrm{C} 43$ & $0.079(5)$ & $0.056(5)$ & $0.099(5)$ & $-0.006(4)$ & $0.013(5)$ & $-0.002(5)$ \\
\hline $\mathrm{C} 44$ & $0.073(5)$ & $0.079(6)$ & $0.085(5)$ & $0.018(4)$ & $0.023(4)$ & $-0.003(5)$ \\
\hline $\mathrm{C} 45$ & $0.130(7)$ & $0.089(7)$ & $0.152(7)$ & $-0.050(6)$ & $-0.026(6)$ & $0.037(6)$ \\
\hline $\mathrm{C} 46$ & $0.087(5)$ & $0.067(5)$ & $0.096(5)$ & $0.002(5)$ & $0.025(5)$ & $-0.015(5)$ \\
\hline $\mathrm{C} 47$ & $0.103(6)$ & $0.123(8)$ & $0.127(7)$ & $0.027(6)$ & $0.012(6)$ & $0.011(7)$ \\
\hline $\mathrm{C} 48$ & $0.106(7)$ & $0.180(11)$ & $0.111(7)$ & $0.042(7)$ & $0.001(6)$ & $-0.025(7)$ \\
\hline C49 & $0.116(7)$ & $0.079(6)$ & $0.120(7)$ & $-0.022(6)$ & $-0.009(6)$ & $-0.016(6)$ \\
\hline C50 & $0.172(9)$ & $0.100(7)$ & $0.157(9)$ & $0.023(7)$ & $-0.073(8)$ & $-0.039(7)$ \\
\hline $\mathrm{C} 51$ & $0.106(6)$ & $0.118(8)$ & $0.131(7)$ & $0.003(7)$ & $-0.009(6)$ & $-0.012(7)$ \\
\hline C52 & $0.150(9)$ & $0.121(9)$ & $0.204(10)$ & $-0.049(8)$ & $-0.091(9)$ & $0.017(8)$ \\
\hline $\mathrm{C} 53$ & $0.112(7)$ & $0.158(10)$ & $0.160(9)$ & $-0.032(8)$ & $-0.021(7)$ & $-0.004(9)$ \\
\hline C54 & $0.143(11)$ & $0.247(19)$ & $0.209(13)$ & $0.001(12)$ & $-0.051(11)$ & $0.096(14)$ \\
\hline C55 & $0.24(2)$ & $0.30(3)$ & $0.70(5)$ & $-0.11(2)$ & $-0.14(3)$ & $0.32(3)$ \\
\hline C56 & $0.140(9)$ & $0.31(2)$ & $0.150(10)$ & $0.009(12)$ & $-0.034(8)$ & $-0.006(13)$ \\
\hline
\end{tabular}


Table 4. BondLengths for non-hydrogen atoms.

\begin{tabular}{llll}
\hline Atoms & Length & Atoms & Length \\
\hline C1-C2 & $1.344(19)$ & C30-C31 & $1.465(11)$ \\
C2-C3 & $1.524(15)$ & C30-C35 & $1.516(11)$ \\
C3-C4 & $1.351(15)$ & C31-C32 & $1.535(10)$ \\
C4-C5 & $1.525(15)$ & C32-C33 & $1.531(10)$ \\
C5-O6 & $1.410(11)$ & C33-C34 & $1.514(10)$ \\
O6-C7 & $1.393(10)$ & C33-C36 & $1.528(10)$ \\
C7-C12 & $1.319(12)$ & C33-C37 & $1.538(9)$ \\
C7-C8 & $1.364(13)$ & C34-C40 & $1.317(11)$ \\
C8-C9 & $1.349(13)$ & C34-C35 & $1.518(10)$ \\
C9-C10 & $1.407(12)$ & C37-C41 & $1.498(9)$ \\
C10-C11 & $1.367(12)$ & C37-C38 & $1.560(9)$ \\
C10-C13 & $1.426(12)$ & C38-C44 & $1.500(8)$ \\
C11-C12 & $1.433(12)$ & C38-C39 & $1.525(10)$ \\
C12-C14 & $1.204(11)$ & C39-C40 & $1.483(10)$ \\
C14-C15 & $1.404(12)$ & C41-C42 & $1.538(9)$ \\
C15-C16 & $1.366(13)$ & C42-C43 & $1.505(10)$ \\
C15-C20 & $1.408(13)$ & C43-C45 & $1.517(10)$ \\
C16-C17 & $1.410(12)$ & C43-C44 & $1.521(9)$ \\
C17-C18 & $1.364(13)$ & C43-C46 & $1.555(9)$ \\
C18-C19 & $1.317(14)$ & C44-C48 & $1.532(10)$ \\
C18-O21 & $1.378(10)$ & C46-C49 & $1.513(9)$ \\
C19-C20 & $1.357(15)$ & C46-C47 & $1.526(10)$ \\
O21-C22 & $1.370(12)$ & C47-C48 & $1.508(10)$ \\
C22-C23 & $1.528(12)$ & C49-C51 & $1.499(11)$ \\
C23-C24 & $1.451(15)$ & C49-C50 & $1.538(12)$ \\
C24-C25 & $1.564(12)$ & C51-C52 & $1.512(10)$ \\
C25-C26 & $1.478(14)$ & C52-C53 & $1.513(13)$ \\
C26-C27 & $1.471(14)$ & C53-C54 & $1.467(15)$ \\
C27-O28 & $1.130(15)$ & C54-C55 & $1.29(2)$ \\
C27-O29 & $1.205(14)$ & C54-C56 & $1.50(2)$ \\
O29-C30 & $1.443(9)$ & & \\
\hline & & & \\
\hline
\end{tabular}

[6]) by the method of full-matrix least-squares on $F^{2}$. Initial refinement was done with isotropic temperature factors for the non-hydrogen atoms which saturated at $R=0.1548$. At this stage the hydrogens were placed at calculated positions and refinement was carried out with anisotropic temperature factors for the non-hydrogens. The hydrogens were treated as riding on the parent atom. The final cycle of the refinement was carried out with 4308 reflections with 566 parameters which saturated at $R=0.0772$. The largest peak and the deepest hole in the final difference Fourier map were 0.154 and $-0.141 \mathrm{e}^{-3}$ respectively. Crystal data and refinement details are given in table 1.

\section{Results and discussion}

The final atomic coordinates with equivalent isotropic temperature factors are given in table 2 for non-hydrogen atoms. The anisotropic thermal parameters are listed in table 3. Tables 4 and 5 give bond lengths and bond angles, respectively, for non-hydrogen atoms. The ORTEP diagram of the molecule with $50 \%$ probability [7] is shown in figure 1. Figures 2-4 show the packing of the molecules down the $a, b$ and $c$ axes, respectively
Table 5. BondAngles for non-hydrogen atoms.

\begin{tabular}{|c|c|c|c|}
\hline Atoms & Angle & Atoms & Angle \\
\hline $\mathrm{C} 1-\mathrm{C} 2-\mathrm{C} 3$ & $117.6(15)$ & C $30-\mathrm{C} 31-\mathrm{C} 32$ & $110.1(8)$ \\
\hline $\mathrm{C} 4-\mathrm{C} 3-\mathrm{C} 2$ & $115.7(13)$ & C31-C32-C33 & $113.6(7)$ \\
\hline $\mathrm{C} 3-\mathrm{C} 4-\mathrm{C} 5$ & $115.8(11)$ & C $34-\mathrm{C} 33-\mathrm{C} 36$ & $108.9(6)$ \\
\hline $\mathrm{O} 6-\mathrm{C} 5-\mathrm{C} 4$ & $107.9(9)$ & C34-C33-C32 & 108.1(7) \\
\hline $\mathrm{C} 7-\mathrm{O} 6-\mathrm{C} 5$ & $115.6(8)$ & C $36-\mathrm{C} 33-\mathrm{C} 32$ & $108.9(7)$ \\
\hline $\mathrm{C} 12-\mathrm{C} 7-\mathrm{C} 8$ & $120.1(9)$ & C34-C33-C37 & $110.6(6)$ \\
\hline $\mathrm{C} 12-\mathrm{C} 7-\mathrm{O} 6$ & $125.5(10)$ & C36-C33-C37 & $111.3(6)$ \\
\hline $\mathrm{C} 8-\mathrm{C} 7-\mathrm{O} 6$ & $114.4(9)$ & C $32-C 33-C 37$ & $108.9(6)$ \\
\hline $\mathrm{C} 9-\mathrm{C} 8-\mathrm{C} 7$ & $122.9(10)$ & C40-C34-C33 & $123.1(8)$ \\
\hline C8-C9-C10 & $118.7(11)$ & C40-C34-C35 & $120.3(8)$ \\
\hline C11-C10-C9 & $118.7(9)$ & C33-C34-C35 & $116.6(8)$ \\
\hline C11-C10-C13 & $123.7(10)$ & C $30-\mathrm{C} 35-\mathrm{C} 34$ & $110.1(7)$ \\
\hline C9-C10-C13 & $117.6(11)$ & $\mathrm{C} 41-\mathrm{C} 37-\mathrm{C} 33$ & $114.7(6)$ \\
\hline $\mathrm{C} 10-\mathrm{C} 11-\mathrm{C} 12$ & $119.9(9)$ & C41-C37-C38 & $111.3(6)$ \\
\hline $\mathrm{C} 7-\mathrm{C} 12-\mathrm{C} 11$ & $119.7(9)$ & C33-C37-C38 & $112.0(6)$ \\
\hline $\mathrm{C} 14-\mathrm{C} 13-\mathrm{C} 10$ & $177.4(10)$ & C44-C38-C39 & 111.4(7) \\
\hline $\mathrm{C} 13-\mathrm{C} 14-\mathrm{C} 15$ & $179.3(11)$ & C44-C38-C37 & $108.6(6)$ \\
\hline C16-C15-C14 & $118.2(11)$ & C39-C38-C37 & $108.4(6)$ \\
\hline $\mathrm{C} 16-\mathrm{C} 15-\mathrm{C} 20$ & $116.7(9)$ & C40-C39-C38 & $113.3(8)$ \\
\hline $\mathrm{C} 14-\mathrm{C} 15-\mathrm{C} 20$ & $124.8(12)$ & C34-C40-C39 & $124.9(9)$ \\
\hline $\mathrm{C} 15-\mathrm{C} 16-\mathrm{C} 17$ & $121.7(10)$ & C37-C41-C42 & $115.7(6)$ \\
\hline C18-C17-C16 & $117.8(10)$ & $\mathrm{C} 43-\mathrm{C} 42-\mathrm{C} 41$ & $111.1(7)$ \\
\hline C19-C18-C17 & $121.6(10)$ & $\mathrm{C} 42-\mathrm{C} 43-\mathrm{C} 45$ & $109.0(7)$ \\
\hline $\mathrm{C} 19-\mathrm{C} 18-\mathrm{O} 21$ & $112.8(11)$ & $\mathrm{C} 42-\mathrm{C} 43-\mathrm{C} 44$ & $106.0(6)$ \\
\hline $\mathrm{C} 17-\mathrm{C} 18-\mathrm{O} 21$ & $125.5(11)$ & $\mathrm{C} 45-\mathrm{C} 43-\mathrm{C} 44$ & $115.2(7)$ \\
\hline C18-C19-C20 & $121.5(11)$ & $\mathrm{C} 42-\mathrm{C} 43-\mathrm{C} 46$ & $116.9(6)$ \\
\hline C19-C20-C15 & $120.7(11)$ & C45-C43-C46 & $110.2(6)$ \\
\hline $\mathrm{C} 22-\mathrm{O} 21-\mathrm{C} 18$ & $119.2(8)$ & C44-C43-C46 & $99.4(6)$ \\
\hline $\mathrm{O} 21-\mathrm{C} 22-\mathrm{C} 23$ & $110.9(10)$ & C38-C44-C43 & $115.9(6)$ \\
\hline $\mathrm{C} 24-\mathrm{C} 23-\mathrm{C} 22$ & $109.8(11)$ & C38-C44-C48 & $116.5(6)$ \\
\hline $\mathrm{C} 23-\mathrm{C} 24-\mathrm{C} 25$ & $115.8(11)$ & $\mathrm{C} 43-\mathrm{C} 44-\mathrm{C} 48$ & $104.2(6)$ \\
\hline $\mathrm{C} 26-\mathrm{C} 25-\mathrm{C} 24$ & $114.5(10)$ & C49-C46-C47 & $112.3(7)$ \\
\hline $\mathrm{C} 25-\mathrm{C} 26-\mathrm{C} 27$ & $114.3(10)$ & C49-C46-C43 & $121.3(7)$ \\
\hline $\mathrm{O} 28-\mathrm{C} 27-\mathrm{O} 29$ & $120.3(13)$ & C47-C46-C43 & $103.4(6)$ \\
\hline $\mathrm{O} 28-\mathrm{C} 27-\mathrm{C} 26$ & $122.2(16)$ & $\mathrm{C} 48-\mathrm{C} 47-\mathrm{C} 46$ & $108.3(6)$ \\
\hline $\mathrm{O} 29-\mathrm{C} 27-\mathrm{C} 26$ & $117.5(14)$ & C47-C48-C44 & $103.0(6)$ \\
\hline $\mathrm{C} 27-\mathrm{O} 29-\mathrm{C} 30$ & $121.4(10)$ & C51-C49-C46 & $111.6(7)$ \\
\hline O29-C30-C31 & $107.3(8)$ & C51-C49-C50 & $110.0(7)$ \\
\hline O29-C30-C35 & $108.7(7)$ & C46-C49-C50 & $112.4(7)$ \\
\hline C $31-C 30-C 35$ & $110.2(7)$ & C49-C51-C52 & $117.9(8)$ \\
\hline C51-C52-C53 & $113.1(9)$ & C55-C54-C56 & $111.2(15)$ \\
\hline C54-C53-C52 & $119.4(11)$ & C53-C54-C56 & $108.8(15)$ \\
\hline C55-C54-C53 & $108.6(16)$ & & \\
\hline
\end{tabular}

[8]. The bond distances and angles are in good agreement with the values for compounds containing phenyl and cholesterol moieties [9-11]. DMT5 crystallizes in the $P 2_{1}$ space group, as in the case of a similar dimesogen, DM, 4-(cholesteroxycarbony 1)pentyloxy-4' - heptyloxycarbonylbiphenyl [12]. This indicates that the dimesogens should conform to either $P 2_{1}$ or $P 2_{1} 2_{1} 2_{1}$ space groups for them to exhibit a chiral mesophase. Intermolecular hydrogen bonds of the type $\mathrm{CH} \ldots \mathrm{O}$ are present, viz. C3-H3A ... O6, with length 2.802(2) $\AA$ and C24-H24B ... O21 with length 2.898(7) $\AA$. The two phenyl rings are independently planar and are almost parallel as the dihedral angle between them is $4^{\circ}$. The 


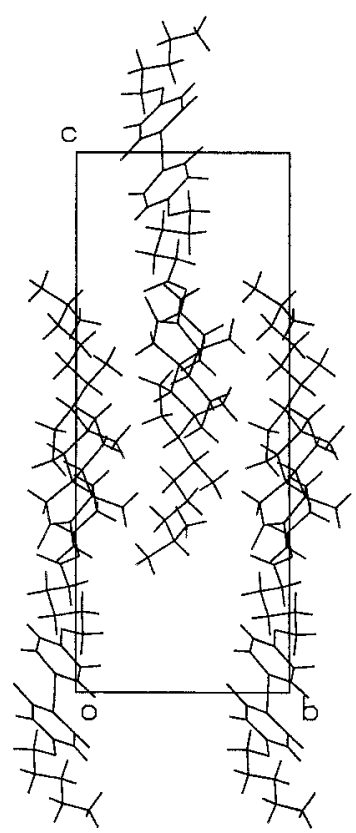

Figure 2. Packing of DMT5 molecules down the $a$-axis.

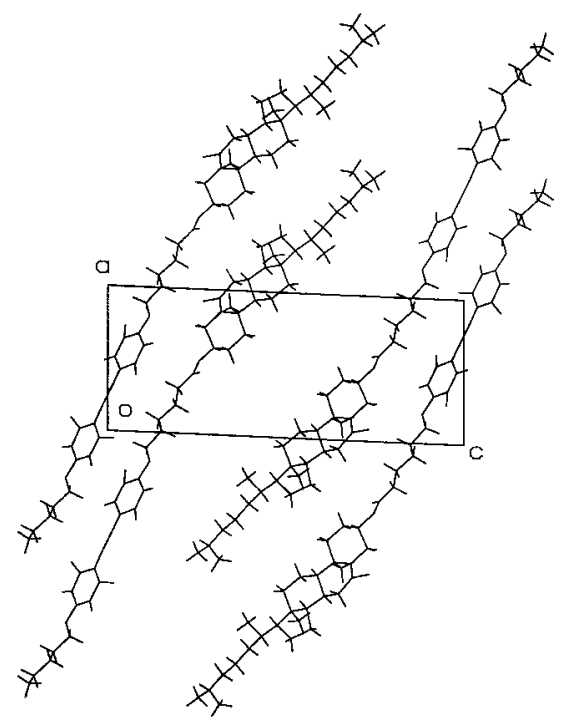

Figure 3. Packing of DMT5 molecules down the $b$-axis. fused rings of the cholesterol moiety are almost perpendicular to the phenyl rings. The dihedral angles between the planes of the fused rings of the cholesterol moiety and the phenyl rings are $87.7^{\circ}$ and $88.3^{\circ}$. This is in contrast to the structure of DM in reference [12] where the angles are $18.9^{\circ}$ and $19.75^{\circ}$. Further the molecule is extended, unlike the molecule of DM. The deviations can be attributed to the presence of a triple bond between the phenyl groups which reduces the flexibility. The structure also has many intermolecular contacts closer than van der Waals' radii which stabilize the structure. The greater flexibility of DM results in the occurrence of multiple mesophases, unlike in this structure where only a single mesophase is exhibited.

The authors would like to thank DST, Government of India, for financial assistance under project No. $\mathrm{SP} / \mathrm{I} 2 / \mathrm{FOO} / 93$. The authors would like to thank Prof. S. Chandrasekhar for encouragement and Prof. P. S. Zacharias for data collection.

\section{References}

[1] For a brief but recent review on dimers see: 1998, Handbook of Liquid Crystals, Vol 2B, edited by D. Demus, J. Goodby, G. W. Gray, and H. W. Speiss, p. 801.

[2] Attard, G. S., Date, R. W., Imrie, C. T., Luckhurst, G. R., Roskilly, S. J., Seddon, J. M., and TAYLOR, L., 1994, Liq. Cryst., 16, 529.

[3] Hardouin, F., Achard, M. F., Jin, J. I., Shin, J. W., and Yun, Y. K., 1994, J. Phys. II, 4, 627.

[4] Yelamaggad, C. V., 1999, Mol. Cryst. liq. Cryst. (in the press).

[5] SHELdRICK, G. M., 1997, SHELXS-97, University of Göttingen, Germany.

[6] SHELDRICK, G. M., 1997, SHELXL-97, University of Göttingen, Germany.

[7] SpeK, A. L., 1990, Acta Cryst., A46, C-34.

[8] Sawzik, P., and Craven, B. M., 1980, Acta Cryst., B36, 215.

[9] Sawzik, P., and Craven, B. M., 1980, Acta Cryst., B36, 3027.

[10] Manisekaran, T., Varghese, B., and Shashidhara Prasad, J., 1996, Mol. Cryst. liq. Cryst., 289, 221.

[11] Manisekaran, T., Bamezai, R. K., Sharma, N. K., and Shashidhara Prasad, J., 1997, Liq. Cryst., 27, 597.

[12] Shashidhara Prasad, J., Sridhar, M. A., and Surendranath, V., 1999, Liq. Cryst. (in the press).

Figure 4. Packing of DMT5 molecules down the $c$-axis.

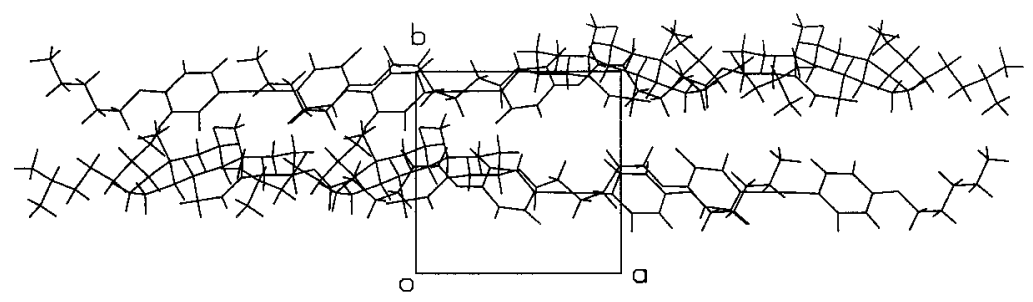

